\title{
Polyharmonic Hardy Spaces on the Complexified Annulus and Error Estimates of Cubature Formulas
}

\author{
Ognyan Kounchev and Hermann Render
}

To the memory of Professor Werner Haußmann

\begin{abstract}
The present paper has a twofold contribution: first, we introduce a new concept of Hardy spaces on a multidimensional complexified annular domain which is closely related to the annulus of the Klein-Dirac quadric important in Conformal Quantum Field Theory. Secondly, for functions in these Hardy spaces, we provide error estimate for the polyharmonic Gauß-Jacobi cubature formulas, which have been introduced in previous papers.
\end{abstract}

Mathematics Subject Classification (2000). Primary 65D30, 32A35; Secondary 41A55.

Keywords. Hardy space, Numerical Integration, Cubature formulas, Error estimate.

\section{Introduction}

In the classical one-dimensional theory of quadrature formulas there are different approaches to the estimation of the quadrature formulas of Gauss type. The first approach established already by A. Markov estimates the error of a quadrature formula for differentiable functions in $C^{N}(I)$ defined on the interval $I$ by means of its $N$-th derivative, cf. Krylov 31, chapter 7.1, or Davis [10, p. 344]. However this approach is usually not very practical beyond derivatives of order five, see 52. The second approach estimates the error for certain classes of functions $f$ which are analytic on some open set $D$ in $\mathbb{C}$ containing the interval $I$, cf. [11, chapter 4.6, see also [31, chapter 12.2.

The first-named author thanks the Alexander von Humboldt Foundation and Project DO02-275 with Bulgarian NSF. 
Approximation of integrals in the multivariate case is a much more difficult task. In Numerical Analysis, instead of quadrature formula the notion of cubature formula is often used, see [47, [51, [48] and the recent survey 9. In contrast to the univariate case there is no satisfactory error analysis available in the multivariate case, cf. [51, 6], and part 4 in the last Russian edition of the classical monograph [31. In passing we mention that the area of quadrature formulas for harmonic functions in which Werner Haußmann has worked, are an interesting multidimensional alternative and we refer to [2], 16] on this topic.

\subsection{Preliminaries}

In [23] and 21] we have introduced a new multivariate cubature formulae $C_{N}(f)$ depending on a parameter $N \in \mathbb{N}$ which approximates the integral

$$
\int_{A_{a, b}} f(x) d \mu(x)
$$

for continuous functions $f: A_{a, b} \rightarrow \mathbb{C}$ defined on the annular region

$$
A_{a, b}=\left\{x \in \mathbb{R}^{d}: a<|x|<b\right\},
$$

where $|x|=\sqrt{x_{1}^{2}+\ldots .+x_{d}^{2}}$ is the euclidean norm of $x=\left(x_{1}, \ldots, x_{d}\right) \in \mathbb{R}^{d}$. In [23], 21], we have considered a special class of signed measures $\mu$ with support in $A_{a, b}$, the so-called pseudo-positive measures (see definition below) however this formula is easy to extend to almost all signed measure. The exact definition of the cubature formula $C_{N}(f)$ will be explained in Section 7. One aim of this paper is to provide an error analysis for a class of functions on the annular region $A_{a, b}$ which exhibit a certain type of analytical behavior.

In order to give the reader the needed mathematical background we have to recall some terminology. Let

$$
\mathbb{S}^{d-1}:=\left\{x \in \mathbb{R}^{d}:|x|=1\right\}
$$

be the unit sphere endowed with the rotation invariant measure $d \theta$. We shall write $x \in \mathbb{R}^{d}$ in spherical coordinates $x=r \theta$ with $\theta \in \mathbb{S}^{d-1}$. Let $\mathcal{H}_{k}\left(\mathbb{R}^{d}\right)$ be the set of all harmonic homogeneous complex-valued polynomials of degree $k$. Then $f \in \mathcal{H}_{k}\left(\mathbb{R}^{d}\right)$ is called a solid harmonic and the restriction of $f$ to $\mathbb{S}^{d-1}$ a spherical harmonic of degree $k$ and we set

$$
a_{k}:=\operatorname{dim} \mathcal{H}_{k}\left(\mathbb{R}^{d}\right),
$$

see [50, 45], 1], 20] for details. Throughout the paper we shall assume

$$
Y_{k, \ell}: \mathbb{R}^{d} \rightarrow \mathbb{R}, \ell=1, \ldots, a_{k},
$$

is an orthonormal basis of $\mathcal{H}_{k}\left(\mathbb{R}^{d}\right)$ with respect to the scalar product

$$
\langle f, g\rangle_{\mathbb{S}^{d-1}}:=\int_{\mathbb{S}^{d-1}} f(\theta) \overline{g(\theta)} d \theta .
$$

We shall often use the trivial identity $Y_{k, \ell}(x)=r^{k} Y_{k \ell}(\theta)$ for $x=r \theta$. The class of pseudo-positive measures used for our cubature formula $C_{N}(f)$ is 
now defined in the following way: a signed measure $\mu$ with support in $A_{a, b} \subset$ $\mathbb{R}^{d}$ is pseudo-positive with respect to the orthonormal basis $Y_{k, \ell}, \ell=1, \ldots, a_{k}$, $k \in \mathbb{N}_{0}$ if the inequality

$$
\int_{\mathbb{R}^{d}} h(|x|) Y_{k, \ell}(x) d \mu(x) \geq 0
$$

holds for every non-negative continuous function $h:[a, b] \rightarrow[0, \infty)$ and for all $k \in \mathbb{N}_{0}, \ell=1,2, \ldots, a_{k}$. Let us note that every signed measure $d \mu$ with bounded variation may be represented (non-uniquely) as a difference of two pseudo-positive measures. We refer to [23] for instructive examples of pseudopositive measures.

The cubature formula $C_{N}(f)$ approximating the integral (1.1) is based on the Laplace-Fourier series of the continuous function $f: A_{a, b} \rightarrow \mathbb{C}$, defined by the formal expansion

$$
f(r \theta)=\sum_{k=0}^{\infty} \sum_{\ell=1}^{a_{k}} f_{k, \ell}(r) Y_{k, \ell}(\theta)
$$

where the Laplace-Fourier coefficient $f_{k, \ell}(r)$ is defined by

$$
f_{k, \ell}(r)=\int_{\mathbb{S}^{d-1}} f(r \theta) Y_{k, \ell}(\theta) d \theta
$$

for any positive real number $r$ with $a<r<b$ and $a_{k}$ is defined in (1.3). There is a strong interplay between algebraic and analytic properties of the function $f$ and those of the Laplace-Fourier coefficients $f_{k, \ell}$. For example, if $f(x)$ is a polynomial in the variable $x=\left(x_{1}, \ldots, x_{d}\right)$ then the Laplace-Fourier coefficient $f_{k, \ell}$ is of the form $f_{k, \ell}(r)=r^{k} p_{k, \ell}\left(r^{2}\right)$ where $p_{k, \ell}$ is a univariate polynomial, see e.g. in [50] or [47. Hence, the Laplace-Fourier series (1.6) of a polynomial $f(x)$ is equal to

$$
f(x)=\sum_{k=0}^{\operatorname{deg} f} \sum_{\ell=1}^{a_{k}} p_{k, \ell}\left(|x|^{2}\right) Y_{k, \ell}(x)
$$

where $\operatorname{deg} f$ is the total degree of $f$ and $p_{k, \ell}$ is a univariate polynomial of degree $\leq \operatorname{deg} f-k$. This representation is often called the Gauss representation. A similar formula is valid for a much larger class of functions. Let us recall that a function $f: G \rightarrow \mathbb{C}$ defined on an open set $G$ in $\mathbb{R}^{d}$ is called polyharmonic of order $N$ if $f$ is $2 N$ times continuously differentiable and

$$
\Delta^{N} u(x)=0
$$

for all $x \in G$ where $\Delta=\frac{\partial^{2}}{\partial x_{1}^{2}}+\ldots+\frac{\partial^{2}}{\partial x_{d}^{2}}$ is the Laplace operator and $\Delta^{N}$ the $N$-th iterate of $\Delta$. The theorem of Almansi states that for a polyharmonic function $f$ of order $N$ defined on the ball $B_{R}=\left\{x \in \mathbb{R}^{d}:|x|<R\right\}$ there exist univariate polynomials $p_{k, \ell}(r)$ of degree $\leq N-1$ such that

$$
f(x)=\sum_{k=0}^{\infty} \sum_{\ell=1}^{a_{k}} p_{k, \ell}\left(|x|^{2}\right) Y_{k, \ell}(x)
$$


where convergence of the sum is uniform on compact subsets of $B_{R}$, see e.g. [7], 4, 3].

In this paper an important role is played by the representation of polyharmonic functions on the annular region $A_{a, b}$ developed by Vekua and Sobolev (see 54, 47): It is especially simple if the dimension $d$ of the euclidean space $\mathbb{R}^{d}$ is odd. Then for any polyharmonic function $f$ of order $N$ defined on the annular region $A_{a, b}$ there exist univariate $\widetilde{u}_{k, \ell}(\cdot), \widetilde{\widetilde{u}}_{k, \ell}(\cdot)$ polynomials of degree $\leq N-1$ such that

$$
u(x)=\sum_{k=0}^{\infty} \sum_{\ell=1}^{a_{k}}\left(\widetilde{u}_{k, \ell}\left(|x|^{2}\right) r^{k}+\widetilde{\widetilde{u}}_{k, \ell}\left(|x|^{2}\right) r^{-d-k+2}\right) Y_{k, \ell}(\theta)
$$

where convergence of the sum is uniform on compact subsets of $A_{a, b}$. An equivalent way to express this is to write

$$
u(r \theta)=\sum_{k, \ell}\left(\sum_{j=0}^{N-1} c_{k, \ell ; j} r^{k+2 j}+\sum_{j=0}^{N-1} d_{k, \ell ; j} r^{-d-k+2+2 j}\right) Y_{k, \ell}(\theta) .
$$

In particular, one obtains a representation of the polynomials if all $d_{k, \ell ; j}$ are zero.

\subsection{Complexification of the annulus and the annulus of the Klein-Dirac quadric}

We want to study analytical extensions of functions $f$ defined on the annular region using the Laplace-Fourier series. Instead of working with functions $f$ which are a priori analytically extendible to a fixed domain $U$ in the complex space $\mathbb{C}^{d}$ we shall require only that we can extend the function $x=r \theta \longmapsto$ $f(r \theta)$ to an analytic function $z \theta \longmapsto f(z \theta)$, so we only complexify the radial variable $r$ to a complex variable $z$. Then one should expect from (1.7) that the Laplace-Fourier coefficient $f_{k, \ell}(r)$ extends to an analytic function of one variable.

To make things more precise let us introduce the basis functions

$$
R_{j}(r):=\left\{\begin{array}{cc}
r^{2 j+k} & \text { for } j=0,1, \ldots, N-1 \\
r^{-d-k+2+2(j-N)} & \text { for } j=N, N+1, \ldots, 2 N-1 .
\end{array}\right.
$$

By representation (1.11) we see that the functions

$$
\left\{R_{j}(r) Y_{k, \ell}(\theta), \quad \text { for } k \geq 0, \ell=1,2, \ldots, a_{k}, j=0,1, \ldots, 2 N-1\right\}
$$

form a basis for the functions polyharmonic of order $N$ in the annulus $A_{a, b}$ 1

For every fixed $k$ we introduce the following convenient notation for the set of indices to be used further:

$\mathcal{R}_{k}:=\{j \in \mathbb{Z}: j=k+2 m$, or $j=-d-k+2+2 m$ for an integer $m \geq 0\}$.

As said above, the main feature of our approach is to work with a complexification of the functions $f$ defined in the annulus $A_{a, b}$ of the form $f(z \theta)$.

\footnotetext{
${ }^{1}$ The functions $R_{j}$ for $j=0,1, . ., N-1$ are called polyharmonic Kelvin transforms of the functions $R_{N+j}$, cf. [3] p. 6 .
} 
For polyharmonic functions $f$ representation (1.11) provides a direct description of this complexification by considering the complexified basis functions $R_{j}(z)$; we consider the linear space generated by the functions:

$$
V_{k, d}:=\operatorname{span}\left\{\left\{z^{2 j+k}\right\}_{j=0}^{\infty},\left\{z^{-d-k+2+2 j}\right\}_{j=0}^{\infty}\right\}
$$

Now in these new notations, a generic element in the space $V_{k, d}$ has the form $\widetilde{u}_{k, \ell}\left(z^{2}\right) z^{k}+\widetilde{\widetilde{u}}_{k, \ell}\left(z^{2}\right) z^{-d-k+2}$ where $\widetilde{u}_{k, \ell}(\cdot)$ and $\widetilde{\widetilde{u}}_{k, \ell}(\cdot)$ are polynomials. Obviously, $V_{k, d} \subset \mathcal{H}\left(\mathbb{A}_{a, b}\right), V_{k, d} \subset \mathcal{H}\left(\mathbb{A}_{0, b}\right)$, and $V_{k, d} \subset \mathcal{H}(\mathbb{C} \backslash\{0\})$ where $\mathcal{H}(\Omega)$ denotes the space of all analytic functions in a domain $\Omega \subset \mathbb{C}$.

Thus, we have come naturally to the next objective of the present research, the Function theory in the space $\mathbb{C} \times \mathbb{S}^{n-1}$. The main interest in the present paper is devoted to the complexified annulus $\mathcal{A}_{a, b}$ given by

$$
\mathcal{A}_{a, b}:=\left\{(z, \theta): a<|z|<b, \theta \in \mathbb{S}^{d-1}\right\}=\mathbb{A}_{a, b} \times \mathbb{S}^{d-1}
$$

here $\mathbb{A}_{a, b}$ is the annulus in $\mathbb{C}$,

$$
\mathbb{A}_{a, b}:=\{z \in \mathbb{C}: a<|z|<b\} .
$$

Our main novelty will be a multivariate generalization of $H^{2}\left(\mathbb{A}_{a, b}\right)$ called (polyharmonic) Hardy space on the annulus $\mathcal{A}_{a, b}$ to be introduced in Definition 4.5. We will denote this space by $H_{L}^{2}\left(\mathcal{A}_{a, b}\right)$ since it depends on a parameter $L>1$. The name polyharmonic comes from the fact that $H_{L}^{2}\left(\mathcal{A}_{a, b}\right)$ is obtained as a limit of the complexifications of the polyharmonic functions in the annulus (1.11), namely, of the space:

$$
\mathcal{V}:=\operatorname{span}\left\{z^{k+2 j} Y_{k, \ell}(\theta), z^{-d-k+2+2 j} Y_{k, \ell}(\theta): k, j \geq 0, \ell=1,2, \ldots, a_{k}\right\}
$$

where $z \in \mathbb{C}$ and $\theta \in \mathbb{S}^{d-1}$. Clearly, the elements of $\mathcal{V}$ are finite sums of the type

$$
u(z, \theta)=\sum_{k, \ell}\left(\widetilde{u}_{k, \ell}\left(z^{2}\right) z^{k}+\widetilde{\widetilde{u}}_{k, \ell}\left(z^{2}\right) z^{-d-k+2}\right) Y_{k, \ell}(\theta),
$$

where $\widetilde{u}_{k, \ell}(\cdot)$ and $\widetilde{\widetilde{u}}_{k, \ell}(\cdot)$ are algebraic polynomials. In view of the Polyharmonic Paradigm announced in [20, the space $H_{L}^{2}\left(\mathcal{A}_{a, b}\right)$ generalizes the classical Hardy spaces which are obtained as limits of (Laurent) polynomials, where the degree of a polynomial is replaced by a degree of polyharmonicity.

Let us summarize briefly our objectives: The main approach to the polyharmonic Hardy space $H_{L}^{2}\left(\mathcal{A}_{a, b}\right)$ is to generate it by the complexified finite sums (1.17), by introducing an appropriate inner product (and norm). This Hardy space will be a Hilbert space and we will provide a Cauchy type kernel, which is the analog and a generalization to the Hua-Aronszajn kernel in the ball (cf. 3, p. 125, Corollary 1.1). Let us note that the last is a multidimensional generalization of the classical Cauchy kernel $\frac{1}{z}$ from Complex Analysis (various about Cauchy kernels see in [18, [28]). 
Remark 1.1. Our results are closely related to the Function theory on the Klein quadric (called sometimes Klein-Dirac quadric) given by:

$$
\mathrm{KDQ}:=\left\{z \theta: z \in \mathbb{C}, \theta \in \mathbb{S}^{d-1}\right\}=\mathbb{C} \times \mathbb{S}^{d-1} / \mathbb{Z}_{2},
$$

which may be considered as a subset in $\mathbb{C}^{d}$. The factor $\mathbb{Z}_{2}$ means that in the set $\mathbb{C} \times \mathbb{S}^{d-1}$ we identify the elements $(z, \theta) \approx(-z,-\theta)$ and the elements $z \theta=$ $\left(z \theta_{1}, z \theta_{2}, \ldots, z \theta_{d}\right)$ of KDQ are points in $\mathbb{C}^{d}$. The space KDQ is very important for us since a genuine counterpart of the present Hardy spaces exists only on the ball $\mathbb{D} \times \mathbb{S}^{n-1} / \mathbb{Z}_{2}$ of KDQ (and not on the ball $\mathbb{D} \times \mathbb{S}^{n-1}$ ) which is due to the fact that the polyharmonic functions in the ball in $\mathbb{R}^{d}$ have representation of the type (1.11) with terms $z^{k+2 j} Y_{k, \ell}(\theta)=z^{2 j} Y_{k, \ell}(z \theta)$ depending on $z \theta$. In a forthcoming research [26, 24] we study Hardy spaces on the ball in $\mathrm{KDQ}$. For the Klein quadric the ball $\mathbb{D} \times \mathbb{S}^{n-1} / \mathbb{Z}_{2}$ is the compactified fourdimensional Minkowski space-time $\mathbb{S}^{1} \times \mathbb{S}^{3} / \mathbb{Z}_{2}$. It is hoped that the present research provides a useful framework for CFT by approximating the fields by elements of the Hardy space in the annulus $\mathcal{A}_{a, b}$, see Remark 4.9 below.

The Klein-Dirac quadric KDQ defined in (1.18) for arbitrary dimension $d$, has been originally introduced in a special case by Felix Klein in his Erlangen program in 1870, where he put forth his correspondence between the lines in complex projective 3 -space and a general quadric in projective 5 -space. The physical relevance of the quadric and the relation to the conformal motions of compactified Minkowski space-time had been exploited by Paul Dirac in 1936 12. The Klein-Dirac quadric plays an important role also in Twistor theory, where it is related to the complexified compactified Minkowski space 38. Apparently, the term "Klein-Dirac quadric" for arbitrary dimension $d$, has been coined by the theoretical physicist I. Todorov, cf. e.g. 35, 36. In these references important aspects of the Function theory on the ball in KDQ have been considered in the context of Conformal Quantum Field Theory (CFT). In the context of CFT Laurent expansions appear in a natural way as the field functions in the higher dimensional conformal vertex algebras (using a complex variable parametrization of compactified Minkowski space); see in particular formula (4.43) in [37, as well as the references [35, 36.

The paper is organized as follows: in Section 2 we recall background material about the Hardy space $H^{2}\left(\mathbb{A}_{a, b}\right)$. In Section 3 we define the "component" subspaces $H^{2, k}\left(\mathbb{A}_{a, b}\right)$ of $H^{2}\left(\mathbb{A}_{a, b}\right)$ which will be necessary for the multivariate case. In Section 4 we introduce the polyharmonic Hardy space $H_{L}^{2}\left(\mathcal{A}_{a, b}\right)$ on the complexified annulus $\mathcal{A}_{a, b}$. We prove that it is a Hilbert space, a maximum principle, and infinite-differentiability of the functions in $H_{L}^{2}\left(\mathcal{A}_{a, b}\right)$. In Section 5 we construct a Cauchy type kernel for the component spaces $H^{2, k}\left(\mathbb{A}_{a, b}\right)$ and in Section 6 a Cauchy type kernel for the space $H_{L}^{2}\left(\mathcal{A}_{a, b}\right)$. In Section 7 we prove the main result of the paper, about the error estimate of the polyharmonic Gauß-Jacobi cubature formulas introduced in [21], 23]. 


\section{The inner product and norm in the annulus in $\mathbb{C}$}

Let us recall the classical Hardy space $H^{2}\left(\mathbb{A}_{a, b}\right)$ on the annulus $\mathbb{A}_{a, b}$ and its basic properties, cf. [43], p. 4; see also [5].

Let us remind the usual mean value of a function $f$ defined in $\mathbb{A}_{a, b}$ as

$$
M_{2}(f ; r):=\left\{\frac{1}{2 \pi} \int_{0}^{2 \pi}\left|f\left(r e^{i \varphi}\right)\right|^{2} d \varphi\right\}^{1 / 2}
$$

Definition 2.1. The Hardy space $H^{2}\left(\mathbb{A}_{a, b}\right)$ is defined as the space of all analytic functions $f$ in $\mathbb{A}_{a, b}$ by the condition

$$
\sup _{a<r<b} M_{2}(f ; r)<\infty
$$

Remark 2.2. Recall that by a theorem of Hardy and Riesz the function $\log M_{2}(f ; r)$ is a convex function of $\log r$, hence its maximum is attained either for $r \rightarrow a+$ or for $r \rightarrow b-$; cf. [43], p. 4 .

The following Theorem contains the basic properties of the space $H^{2}\left(\mathbb{A}_{a, b}\right)$, see 43 , p. $7-9$.

Theorem 2.3. Every $f \in H^{2}\left(\mathbb{A}_{a, b}\right)$ is decomposed in $f=g+h$ where $g \in$ $H^{2}\left(\mathbb{D}_{b}\right)$ and $h \in H^{2}\left(\mathbb{C} \backslash \overline{\mathbb{D}_{a}}\right)$, where $\mathbb{D}_{r}$ denotes the disc $\{z \in \mathbb{C}:|z|<r\}$. (Hence, the functions $g$ and $h$ provide the limiting properties to $f$ )

Let $f \in H^{2}\left(\mathbb{A}_{a, b}\right)$ and $f^{*}$ be its limiting function on $\partial \mathbb{A}_{a, b}$ which we denote by $f_{a}^{*}$ on the circle $a \times \mathbb{S}^{1} \subset \mathbb{C}$, and by $f_{b}^{*}$ on the circle $b \times \mathbb{S}^{1} \subset \mathbb{C}$.

(i) If the Laurent expansion of $f$ is given by

$$
f(z)=\sum_{j=-\infty}^{\infty} c_{j} z^{j}
$$

then $f_{a}^{*}, f_{b}^{*} \in L_{2}\left(\mathbb{S}^{1}\right)$ and have Fourier series respectively

$$
\sum_{j} c_{j} a^{j} e^{i j \varphi}, \quad \sum_{j} c_{j} b^{j} e^{i j \varphi} .
$$

Vice versa, to every pair of functions $f_{a}^{*}, f_{b}^{*} \in L_{2}\left(\mathbb{S}^{1}\right)$ having Fourier series (2.4) there corresponds uniquely an $f \in H^{2}\left(\mathbb{A}_{a, b}\right)$ given by (2.3).

(ii) The following Cauchy formula holds:

$$
f(z)=\frac{1}{2 \pi} \int_{0}^{2 \pi} f_{b}^{*}\left(b e^{i \varphi}\right) \frac{b e^{i \varphi}}{\left(b e^{i \varphi}-z\right)} d \varphi-\frac{1}{2 \pi} \int_{0}^{2 \pi} f_{a}^{*}\left(a e^{i \varphi}\right) \frac{a e^{i \varphi}}{\left(a e^{i \varphi}-z\right)} d \varphi
$$

(iii) The following Maximum principle holds:

$$
|f(z)| \leq \frac{2\left(\left\|f_{a}^{*}\right\|_{L_{2}\left(\mathbb{S}^{1}\right)}+\left\|f_{b}^{*}\right\|_{L_{2}\left(\mathbb{S}^{1}\right)}\right)}{\min (1-|z| / b,|z| / a-1)} \quad \text { for all } a<|z|<b .
$$


Remark 2.4. The space $H^{2}\left(\mathbb{A}_{a, b}\right)$ is a Hilbert space with the inner product

$$
\begin{aligned}
& \|f\|_{H^{2}\left(\mathbb{A}_{a, b}\right)}^{2}=\left\|f_{a}^{*}\right\|_{L_{2}}^{2}+\left\|f_{b}^{*}\right\|_{L_{2}}^{2} \\
& \quad=\lim _{r \longrightarrow a} \frac{1}{2 \pi} \int_{0}^{2 \pi} f\left(r e^{i \varphi}\right) \overline{g\left(r e^{i \varphi}\right)} d \varphi+\lim _{r \longrightarrow b} \frac{1}{2 \pi} \int_{0}^{2 \pi} f\left(r e^{i \varphi}\right) \overline{g\left(r e^{i \varphi}\right)} d \varphi
\end{aligned}
$$

cf. 43. Let $f \in H^{2}\left(\mathbb{A}_{a, b}\right)$ and have a Laurent expansion

$$
f(z)=\sum_{j=-\infty}^{\infty} c_{j} z^{j}
$$

Then

$$
\|f\|_{H^{2}\left(\mathbb{A}_{a, b}\right)}^{2}=\sum_{j=-\infty}^{\infty}\left|c_{j}\right|^{2}\left\|z^{j}\right\|_{L_{2}\left(\partial \mathbb{A}_{a, b}\right)}^{2}=2 \pi \sum_{j=-\infty}^{\infty}\left|c_{j}\right|^{2}\left(a^{2 j}+b^{2 j}\right) .
$$

Remark 2.5. In [7] S. Bergman computes the kernel for the annulus, cf. also [29] and [32].

Remark 2.6. The following follows directly from above: For every function $f \in \mathcal{H}\left(\mathbb{A}_{a, b}\right)$ we have the inequalities

$$
\|f\|_{H^{2}\left(\mathbb{A}_{a, b}\right)} \leq 2 \sup _{a<r<b} M_{2}(f ; r) \leq 2\|f\|_{H^{2}\left(\mathbb{A}_{a, b}\right)} .
$$

\section{The component one-dimensional spaces $H^{2, k}$}

For us important role will be played by the so-called "component Hardy spaces" $H^{2, k}\left(\mathbb{A}_{a, b}\right)$ which are relatively simple subspaces of the Hardy space $H^{2}\left(\mathbb{A}_{a, b}\right)$, cf. some classical references on Hardy spaces as [17, [40.

Definition 3.1. The closure of the linear space of analytic functions $V_{k, d}$ defined in (1.14), in the Hardy space $H^{2}\left(\mathbb{A}_{a, b}\right)$ will be denoted by

$$
H^{2, k}=H^{2, k}\left(\mathbb{A}_{a, b}\right)
$$

and will be called component Hardy spaces in the annulus $\mathbb{A}_{a, b}$.

The properties of the space $H^{2, k}\left(\mathbb{A}_{a, b}\right)$ easily follow from those of the usual Hardy spaces $H^{2}\left(\mathbb{A}_{a, b}\right)$ and $H^{2}\left(\mathbb{D}_{b}\right)$.

Proposition 3.2. Let $f \in H^{2, k}\left(\mathbb{A}_{a, b}\right)$.

1. Then $z^{d+k-2} f(z) \in H^{2}\left(\mathbb{D}_{b}\right)$.

2. There exist two uniquely determined functions $f_{1}, f_{2} \in H^{2}\left(\mathbb{D}_{b}\right)$ such that

$$
f(z)=z^{k} f_{1}\left(z^{2}\right)+z^{-d-k+2} f_{2}\left(z^{2}\right) .
$$

3. Let $f^{*}$ (having two parts, $f_{a}^{*}$ and $f_{b}^{*}$ ) be the limiting function for $f$ on $\partial \mathbb{A}_{a, b}$. Then the following expansions hold,

$$
f_{a}^{*}\left(a e^{i \varphi}\right)=\sum_{j \in \mathcal{R}} c_{j} a^{j} e^{i j \varphi}, \quad f_{b}^{*}\left(b e^{i \varphi}\right)=\sum_{j \in \mathcal{R}} c_{j} b^{j} e^{i j \varphi},
$$


where the set $\mathcal{R}$ is defined in (1.13). Vice versa, for every two functions $f_{a}^{*}\left(a e^{i \varphi}\right)$ and $f_{b}^{*}\left(b e^{i \varphi}\right)$ with the above expansions there exists a function $f \in H^{2, k}\left(\mathbb{A}_{a, b}\right)$ having Laurent expansion (2.3) for which $f^{*}$ is a limiting one. holds

4. There exist constants $A, B>0$ such that for every $f \in H^{2, k}\left(\mathbb{A}_{a, b}\right)$

$$
A\|f\|_{H^{2}\left(\mathbb{A}_{a, b}\right)} \leq\left\|f_{1}\right\|_{H^{2}\left(\mathbb{D}_{b}\right)}+\left\|f_{2}\right\|_{H^{2}\left(\mathbb{D}_{b}\right)} \leq B\|f\|_{H^{2}\left(\mathbb{A}_{a, b}\right)} ;
$$

cf. 43, , p. 5 .

\section{Polyharmonic Hardy space $H_{L}^{2}\left(\mathcal{A}_{a, b}\right)$ on the complexified annulus}

\subsection{A motivation}

Now we may define the polyharmonic Hardy space $H_{L}^{2}\left(\mathcal{A}_{a, b}\right)$ on the complexified annulus $\mathcal{A}_{a, b}$ of $\mathbb{R}^{d}$. The most important property of this space will be a Maximum principle, and a related Cauchy type kernel.

Let us explain the problem of finding a proper norm to define the space $H_{L}^{2}\left(\mathcal{A}_{a, b}\right)$. The elements of the space $\mathcal{V}$, which is generating $H_{L}^{2}$, are functions $f(z, \theta)$ having finite expansion

$$
f(z, \theta)=\sum_{k, \ell} f_{k, \ell}(z) Y_{k, \ell}(\theta)=\sum_{k, \ell}\left(\widetilde{f}_{k, \ell}\left(z^{2}\right) z^{k}+\widetilde{\widetilde{f}}_{k, \ell}\left(z^{2}\right) z^{-d-k+2}\right) Y_{k, \ell}(\theta) .
$$

Since all components of this sum are mutually orthogonal in $\theta \in \mathbb{S}^{d-1}$, it would be natural to consider as a candidate the norm

$$
\|f\|_{*}^{2}:=\sum_{k, \ell}\left\|f_{k, \ell}\right\|_{H^{2, k}}^{2} .
$$

However it is easy to see that such a norm does not guarantee that a Cauchy sequence of elements $f_{N}(z \theta), N \geq 1$, will converge uniformly on compacts of $\mathcal{A}_{a, b}$. Indeed, let us consider the following sequence,

$$
u_{N}(z, \theta)=z^{-d-k_{N}+2+2 N} \frac{Y_{k_{N}, 0}(\theta)}{\sqrt[3]{k_{N}}}=\frac{Y_{k_{N}, 0}(\theta)}{\sqrt[3]{k_{N}}}
$$

with $k_{N}=-d+2+2 N$, for $N \geq d$. Obviously, $u_{N} \in \mathcal{V}$ and we have

$$
\left\|u_{N}\right\|_{*}=\frac{1}{\sqrt[3]{k_{N}}} \longrightarrow 0 \quad \text { for } N \longrightarrow \infty
$$

However, as we see below this sequence is even unbounded (for $d \geq 3$ ).

Proposition 4.1. For $d \geq 3$, the sequence of functions $u_{N}$ defined in (4.2) is unbounded.

Proof. First of all, recall that for every spherical harmonic $Y_{k, \ell}(\theta)$ of degree $k \geq 0$ (see (1.4) $)$ we have the estimate

$$
\left|Y_{k, \ell}(\theta)\right| \leq C k^{\frac{d}{2}-1} \quad \text { for all } \theta \in \mathbb{S}^{d-1} .
$$


(cf. 45]). Let us consider $d=3$. We have the following representation of the spherical harmonic which corresponds to the Legendre polynomial $P_{k}(\cdot)$, (cf. 53, Appendix 2, part II ):

$$
Y_{k, 1}(\theta)=\sqrt{\frac{2 k+1}{4 \pi}} P_{k}(\cos \vartheta) \quad \text { for } 0 \leq \vartheta \leq \pi, 0 \leq \varphi \leq 2 \pi
$$

here we have $\theta=(\cos \varphi \sin \vartheta, \sin \varphi \sin \vartheta, \cos \vartheta) \in \mathbb{S}^{2}$, and the usual Legendre polynomials $P_{k}(\cdot)$. Since $P_{k}(1)=1$, it follows that the sequence $u_{N}(z, \theta)$ is unbounded at $\vartheta=0$.

Remark 4.2. An additional observation about the unwished behavior of the sequence $u_{N}(z, \theta)$, which is easy to see for $d=2$ (where $Y_{k, 1}=\cos k \varphi$ and $\left.Y_{k, 2}=\sin k \varphi\right)$ is that derivatives in $\theta$ of $u_{N}(z, \theta)$ contain non-negative powers of $k$.

For that reason a proper norm has to "tame" the behavior of such sequences including their derivatives: we will introduce an inner product and norm which contain weight factors for every component $k$, which "suppress" such unwished behavior and this will guarantee convergence of Cauchy sequences in $H_{L}^{2}\left(\mathcal{A}_{a, b}\right)$ on compact subsets of $\mathcal{A}_{a, b}$.

Remark 4.3. In this context let us remind that a function $f(\theta)$ on $\mathbb{S}^{d-1}$ is real analytic on $\mathbb{S}^{d-1}$ if its Laplace-Fourier expansion $f(\theta)=\sum_{k=0}^{\infty} Y_{k}(\theta)$ (where $\left.Y_{k}(\theta)=\sum_{\ell=1}^{a_{k}} f_{k, \ell} Y_{k, \ell}(\theta)\right)$ satisfies

$$
\left\|Y_{k}(\theta)\right\|<C e^{-\eta k} \quad \text { for } k \geq 1
$$

for some constants $C>0$ and $\eta>0$, cf. [47.

\subsection{The weighted inner product and norm}

Let us introduce the weight factor to be an arbitrary number

$$
L>1
$$

Definition 4.4. Let $f:=\left(f_{k, \ell}\right)_{k, \ell}$ and $g:=\left(g_{k, \ell}\right)_{k, \ell}$ be two sequences of functions with $f_{k, \ell} \in H^{2, k}\left(\mathbb{A}_{a, b}\right)$ and $g_{k, \ell} \in H^{2, k}\left(\mathbb{A}_{a, b}\right)$ for all $k=0,1, \ldots$, and $\ell=1, . ., a_{k}$. We define the following inner product

$$
\langle f, g\rangle_{H_{L}^{2}}:=\sum_{k, \ell}\left\langle f_{k, \ell}, g_{k, \ell}\right\rangle_{H^{2}\left(\mathbb{A}_{a, b}\right)} L^{2 k}
$$

where $\langle,\rangle_{H^{2}\left(\mathbb{A}_{a, b}\right)}$ is the inner product in $H^{2}\left(\mathbb{A}_{a, b}\right)$. 
It is easy to see that $\langle,\rangle_{H^{2}\left(\mathbb{A}_{a, b}\right)}$ satisfies all necessary properties of inner product on a Hilbert space, and the norm is given by

$$
\begin{aligned}
\|f\|_{H_{L}^{2}}^{2} & :=\|f\|_{H_{L}^{2}\left(\mathcal{A}_{a, b}\right)}^{2}:=\sum_{k, \ell}\left\|f_{k, \ell}\right\|_{H^{2}\left(\mathbb{A}_{a, b}\right)}^{2} L^{2 k} \\
& =\left(\frac{1}{2 \pi} \sum_{k, \ell} L^{2 k} \int_{0}^{2 \pi}\left|f_{k, \ell}^{*}\left(a e^{i \varphi}\right)\right|^{2} d \varphi+L^{2 k} \int_{0}^{2 \pi}\left|f_{k, \ell}^{*}\left(b e^{i \varphi}\right)\right|^{2} d \varphi\right)^{1 / 2} .
\end{aligned}
$$

Let us consider the function

$$
f(z, \theta)=\sum_{k, \ell} f_{k, \ell}(z) Y_{k, \ell}(\theta)
$$

with $f_{k, \ell} \in H^{2, k}\left(\mathbb{A}_{a, b}\right)$ and $\|f\|_{H_{L}^{2}}<\infty$. By applying Proposition 2.6] we see that for every $r \in(a, b)$ holds

$$
\begin{aligned}
& \int_{0}^{2 \pi} \int_{\mathbb{S}^{d-1}}\left|\sum_{k, \ell} f_{k, \ell}(z) Y_{k, \ell}(\theta)\right|^{2} d \varphi d \theta \\
& =\sum_{k, \ell} \int_{0}^{2 \pi}\left|f_{k, \ell}\left(r e^{i \varphi}\right)\right|^{2} d \varphi \\
& \leq\|f\|_{H_{L}^{2}}^{2}
\end{aligned}
$$

which shows that $f$ is a function in $L_{2}\left(\mathcal{A}_{a, b}\right)$.

Now we may give the following definition.

Definition 4.5. The polyharmonic Hardy space $H_{L}^{2}\left(\mathcal{A}_{a, b}\right)$ consists of all functions

$$
f(z, \theta)=\sum_{k, \ell} f_{k, \ell}(z) Y_{k, \ell}(\theta)
$$

with $f_{k, \ell} \in H^{2, k}\left(\mathbb{A}_{a, b}\right)$ and satisfying $\|f\|_{H_{L}^{2}}<\infty$.

Let us remark that this definition is completely analogous to the classical case where one takes the Laurent series of the type $\sum_{j=-\infty}^{\infty} a_{j} z^{j}$ which are convergent on compacts in the annulus $\mathbb{A}_{a, b}$.

It is easy to see that there are equivalent representations of the functions in the space $H_{L}^{2}\left(\mathcal{A}_{a, b}\right)$.

Proposition 4.6. Every element $f \in H_{L}^{2}\left(\mathcal{A}_{a, b}\right)$ has the following representation as an infinite series (in $L^{2}-$ sense):

$$
\begin{aligned}
f(z \theta) & =\sum_{k, \ell} f_{k, \ell}(z) Y_{k, \ell}(\theta) \\
& =\sum_{k, \ell} \sum_{j \in \mathcal{R}_{k}} f_{k, \ell ; j} z^{j} Y_{k, \ell}(\theta)
\end{aligned}
$$


where for every index $(k, \ell)$ the function $f_{k, \ell}(z) \in H^{2, k}\left(\mathbb{A}_{a, b}\right)$; the set $\mathcal{R}_{k}$ was introduced in (1.13). The norm of $f$ is given by

$$
\begin{aligned}
\|f\|_{H_{L}^{2}}^{2} & =\sum_{k, \ell}\left(\sum_{j \in \mathcal{R}_{k}}\left|f_{k, \ell ; j}\right|^{2}\left\|z^{j}\right\|_{H^{2}\left(\mathbb{A}_{a, b}\right)}^{2}\right) L^{2 k} \\
& =2 \pi \sum_{k, \ell}\left(\sum_{j \in \mathcal{R}_{k}}\left|f_{k, \ell ; j}\right|^{2}\left(a^{2 j}+b^{2 j}\right)\right) L^{2 k} .
\end{aligned}
$$

The proof is straightforward.

We prove the following fundamental result which contains as its first item that the space $H_{L}^{2}\left(\mathcal{A}_{a, b}\right)$ is a Hilbert one.

Theorem 4.7. 1. The space $H_{L}^{2}\left(\mathcal{A}_{a, b}\right)$ is complete.

2. The following Maximum principle is true: for every compact $K \subset$ $\mathcal{A}_{a, b}$ there exists a constant $C>0$ such that

$$
|f(z, \theta)| \leq \frac{C}{\min (1-|z| / b,|z| / a-1)}\|f\|_{H_{L}^{2}\left(\mathcal{A}_{a, b}\right)} \quad \text { for }(z, \theta) \in K .
$$

3. If $f \in H_{L}^{2}\left(\mathcal{A}_{a, b}\right)$ then $f \in C^{\infty}\left(\mathcal{A}_{a, b}\right)$.

Proof. For the proof of item 1), let $\left\{f^{n}\right\}_{n \geq 1}$ be a Cauchy sequence in $H_{L}^{2}\left(\mathcal{A}_{a, b}\right)$. Then it is clear that for every index $(k, \ell)$ the sequence of functions $\left\{f_{k, \ell}^{n}(z)\right\}_{n \geq 1}$ is a Cauchy sequence in $H^{2}\left(\mathbb{A}_{a, b}\right)$ (even in $\left.H^{2, k}\left(\mathbb{A}_{a, b}\right)\right)$, and as such has a limit $g_{k, \ell}(z) \in H^{2, k}\left(\mathbb{A}_{a, b}\right)$.

We have to show that the function defined by

$$
g(z, \theta)=\sum_{k, \ell} g_{k, \ell}(z) Y_{k, \ell}(\theta)
$$

belongs to $H_{L}^{2}\left(\mathcal{A}_{a, b}\right)$ and is the limit of $\left\{f^{n}\right\}_{n \geq 1}$ there. Let us fix some $\varepsilon>0$. Let us take some $k_{1} \geq 1$. By the Cauchy sequence, we find $n_{1} \geq 1$ such that for all $n, m \geq n_{1}$ we have

$$
\sum_{k=0}^{k_{1}} \sum_{\ell=1}^{a_{k}}\left\|f_{k, \ell}^{n}-f_{k, \ell}^{m}\right\|_{H^{2}\left(\mathbb{A}_{a, b}\right)}^{2} L^{2 k} \leq\left\|f^{n}-f^{m}\right\|_{H_{L}^{2}\left(\mathcal{A}_{a, b}\right)}^{2}<\varepsilon^{2} .
$$

Taking the limit for $m \longrightarrow \infty$ we obtain

$$
\sum_{k=0}^{k_{1}} \sum_{\ell=1}^{a_{k}}\left\|f_{k, \ell}^{n}-g_{k, \ell}\right\|_{H^{2}\left(\mathbb{A}_{a, b}\right)}^{2} L^{2 k}<\varepsilon^{2}
$$

Then taking the limit $k_{1} \longrightarrow \infty$ ends the proof.

2 ). Let us consider an annulus $\mathcal{A}_{a^{\prime}, b^{\prime}}$ with $a<a^{\prime}$ and $b^{\prime}<b$ which satisfies $K \subset \mathcal{A}_{a^{\prime}, b^{\prime}}$. For a function $f$ given by (4.1), by the triangle inequality we obtain for all $(z, \theta) \in K$ the following inequality:

$$
|f(z, \theta)| \leq \sum_{k, \ell}\left|f_{k, \ell}(z)\right|\left|Y_{k, \ell}(\theta)\right| .
$$


By the maximum principle for the annulus, Theorem 2.3, and the estimate for the spherical harmonics (4.3) we obtain

$$
|f(z, \theta)| \leq \frac{2 C}{\min (1-|z| / b,|z| / a-1)} \sum_{k, \ell}\left\|f_{k, \ell}\right\|_{H^{2}\left(\mathbb{A}_{a, b}\right)} k^{\frac{d}{2}-1} .
$$

Further, by applying the Cauchy-Bunyakovsky-Schwarz inequality, for every $(z, \theta) \in \mathcal{A}_{a^{\prime}, b^{\prime}}$ we obtain

$$
\begin{aligned}
& |f(z, \theta)| \\
& \leq \frac{2 C}{\min (1-|z| / b,|z| / a-1)} \sum_{k, \ell} L^{k}\left\|f_{k, \ell}\right\|_{H^{2}\left(\mathbb{A}_{a^{\prime}, b^{\prime}}\right)} k^{\frac{d}{2}-1} L^{-k} \\
& \leq \frac{2 C}{\min (1-|z| / b,|z| / a-1)}\left(\sum_{k, \ell} L^{2 k}\left\|f_{k, \ell}\right\|_{H^{2}\left(\mathbb{A}_{a^{\prime}, b^{\prime}}\right)}^{2}\right)^{1 / 2}\left(\sum_{k, \ell} k^{d-2} L^{-2 k}\right)^{1 / 2} .
\end{aligned}
$$

Since the constant $a_{k}$ in (1.4) is estimated by $a_{k}=O\left(k^{d-2}\right)$ (cf. [50, chapter $4.2)$ it follows that for all $(z, \theta) \in \mathcal{A}_{a^{\prime}, b^{\prime}}$ holds

$$
\begin{aligned}
|f(z, \theta)| & \leq \frac{C_{1}}{\min (1-|z| / b,|z| / a-1)}\|f\|_{H_{L}^{2}\left(\mathcal{A}_{a, b}\right)} \times\left(\sum_{k=0}^{\infty} k^{d-1} k^{d-2} L^{-2 k}\right)^{1 / 2} \\
& \leq \frac{C_{2}}{\min (1-|z| / b,|z| / a-1)}\|f\|_{H_{L}^{2}\left(\mathcal{A}_{a, b}\right)} .
\end{aligned}
$$

This ends the proof.

3. As above we consider $\mathcal{A}_{a^{\prime}, b^{\prime}}$. Let $f \in \mathcal{V}$ have the expansion

$$
f(z, \theta)=\sum_{k, \ell} f_{k, \ell}(z) Y_{k, \ell}(\theta) .
$$

For $(z, \theta) \in \mathcal{A}_{a^{\prime}, b^{\prime}}$ we obtain the estimate

$$
|\partial f(z, \theta) / \partial z| \leq \sum_{k, \ell}\left|f_{k, \ell}^{\prime}(z)\right|\left|Y_{k, \ell}(\theta)\right| .
$$

By the usual Maximum principle in the annulus $\mathbb{A}_{a, b}$ (which is equivalent to continuity of point evaluation operator in $H^{2}\left(\mathbb{A}_{a, b}\right)$, cf. section 17.8 in [40]) we obtain

$$
|\partial f(z, \theta) / \partial z| \leq C \sum_{k, \ell}\left\|f_{k, \ell}\right\|_{H^{2}\left(\mathbb{A}_{a, b}\right)} k^{\frac{d}{2}-1} \quad \text { for all }(z, \theta) \in \mathcal{A}_{a^{\prime}, b^{\prime}},
$$

and the last is convergent as we have seen above. In a similar way, for some derivative $D_{\theta}^{\alpha}$ in $\theta$, where $\alpha$ is a multiindex, we apply the following inequality

$$
\left|D_{\theta}^{\alpha} Y_{k, \ell}(\theta)\right| \leq C_{1} k^{|\alpha|+\frac{d-2}{2}} \quad \text { for } k \geq 1, \theta \in \mathbb{S}^{d-1},
$$

cf. [45], p. 120. This ends the proof.

Remark 4.8. Formula (4.6) is a generalization of the Laurent expansion. 
Remark 4.9. Let us indicate a relation between the Hardy space $H_{L}^{2}\left(\mathcal{A}_{a, b}\right)$ and analogous space on the ball $\mathbb{D} \times \mathbb{S}^{d-1} / \mathbb{Z}_{2}$ of the Klein-Dirac quadric KDQ given in (1.18). Let the function $f \in H_{L}^{2}\left(\mathcal{A}_{a, b}\right)$ be polyharmonic of order $N$ in the sense that in representation (4.1)

$$
f(z, \theta)=\sum_{k, \ell}\left(\widetilde{f}_{k, \ell}\left(z^{2}\right) z^{k}+\widetilde{\widetilde{f}}_{k, \ell}\left(z^{2}\right) z^{-d-k+2}\right) Y_{k, \ell}(\theta)
$$

the functions $\widetilde{f}_{k, \ell}, \widetilde{\widetilde{f}}_{k, \ell}$ are polynomials of degree $N-1$. Then we have the representation

$$
f(z, \theta)=f_{1}(z, \theta)+z^{-d+2} f_{2}(z, \theta)
$$

where the functions

$$
\begin{aligned}
& f_{1}(z, \theta)=\sum_{k, \ell} \widetilde{f}_{k, \ell}\left(z^{2}\right) Y_{k, \ell}(z \theta) \\
& f_{2}(z, \theta)=\sum_{k, \ell} \widetilde{\widetilde{f}}_{k, \ell}\left(z^{2}\right) Y_{k, \ell}\left(\frac{\theta}{z}\right)
\end{aligned}
$$

a obviously defined on the KDQ $\backslash\{0\}$.

\section{The Cauchy type kernel in the component spaces $H^{2, k}\left(\mathbb{A}_{a, b}\right)$}

By the Cauchy formula in the annulus $\mathbb{A}_{a, b}$, for every function $f$ which is analytic in a neighborhood of $\mathbb{A}_{a, b}$ we have:

$$
\begin{aligned}
f(z) & =\frac{1}{2 \pi i} \int_{\Gamma_{b}} \frac{f(\tau) d \tau}{\tau-z}-\frac{1}{2 \pi i} \int_{\Gamma_{a}} \frac{f(\tau) d \tau}{\tau-z} \\
& =\frac{1}{2 \pi i} \int_{\Gamma_{b}}\left(\sum_{j=0}^{\infty} \frac{z^{j}}{\tau^{j+1}}\right) f(\tau) d \tau+\frac{1}{2 \pi i} \int_{\Gamma_{a}}\left(\sum_{j=0}^{\infty} \frac{\tau^{j}}{z^{j+1}}\right) f(\tau) d \tau \\
& =\left.\frac{1}{2 \pi} \int_{0}^{2 \pi}\left(\sum_{j=0}^{\infty} \frac{z^{j}}{\tau^{j}}\right) f(\tau)\right|_{\tau=b e^{i \varphi}} d \varphi \\
& +\left.\frac{1}{2 \pi} \int_{0}^{2 \pi}\left(\sum_{j=0}^{\infty} \frac{\tau^{j+1}}{z^{j+1}}\right) f(\tau)\right|_{\tau=a e^{i \varphi}} d \varphi
\end{aligned}
$$

where we assume that the circular contours $\Gamma_{a}=a \times \mathbb{S}^{1}$ and $\Gamma_{b}=b \times \mathbb{S}^{1}$ have the "positive" (anti-clockwise) orientation and are parametrized by $\tau=a e^{i \varphi}$ and $\tau=b e^{i \varphi}$; we used the notation $\left.g(\tau)\right|_{\tau=b e^{i \varphi}}=g\left(b e^{i \varphi}\right)$. Hence, we select out of the above series only those terms which belong to the space (1.14) and 
we define the following kernels:

$$
\begin{array}{ll}
K_{k}^{1}(z, \tau):=\sum_{j=0}^{\infty}\left(\frac{z}{\tau}\right)^{k+2 j} & \text { for }|\tau|=b \\
K_{k}^{2}(z, \tau):=\sum_{m=-d-k+2+2 j \geq 0}\left(\frac{z}{\tau}\right)^{m} & \text { for }|\tau|=b \\
K_{k}^{3}(z, \tau):=\sum_{m=-d-k+2+2 j<0}\left(\frac{z}{\tau}\right)^{m} & \text { for }|\tau|=a .
\end{array}
$$

It follows that for every $f \in V_{k, d}$ holds

$$
\begin{aligned}
f(z) & =\left.\frac{1}{2 \pi} \int_{0}^{2 \pi}\left(K_{1}(z, \tau)+K_{2}(z, \tau)\right) f(\tau)\right|_{\tau=b e^{i \varphi}} d \varphi \\
& +\left.\frac{1}{2 \pi} \int_{0}^{2 \pi} K_{3}(z, \tau) f(\tau)\right|_{\tau=a e^{i \varphi}} d \varphi
\end{aligned}
$$

Now for every integer $k \geq 0$ we will define the Cauchy type kernel for the annulus $K_{k}$ by setting:

$$
\begin{array}{ll}
K_{k}(z, \tau):=K_{k}^{1}(z, \tau)+K_{k}^{2}(z, \tau) & \text { for }|\tau|=b \\
K_{k}(z, \tau):=K_{k}^{3}(z, \tau) & \text { for }|\tau|=a .
\end{array}
$$

We have the following Proposition which shows that the function $K_{k}$ is the Cauchy type kernel for the space $H^{2, k}\left(\mathbb{A}_{a, b}\right)$ endowed with the inner product in Remark 2.4

Proposition 5.1. Let us denote by $f^{*}$ the limiting value of the function $f \in$ $H^{2, k}\left(\mathbb{A}_{a, b}\right)$ on $\partial \mathbb{A}_{a, b}$. Then for all $z \in \mathbb{A}_{a, b}$ the following Cauchy type formula holds

$$
\begin{aligned}
f(z) & =\left.\frac{1}{2 \pi} \int_{0}^{2 \pi}\left(K_{k}^{1}(z, \tau)+K_{k}^{2}(z, \tau)\right) f^{*}(\tau)\right|_{\tau=b e^{i \varphi}} d \varphi \\
& +\left.\frac{1}{2 \pi} \int_{0}^{2 \pi} K_{k}^{3}(z, \tau) f^{*}(\tau)\right|_{\tau=a e^{i \varphi}} d \varphi \\
& =\frac{1}{2 \pi i} \int_{\partial \mathbb{A}_{a, b}} K_{k}(z, \tau) f^{*}(\tau) \frac{1}{\tau} d \tau \\
& =\left\langle K_{k}(z, \cdot), f(\cdot)\right\rangle_{H^{2}\left(\mathbb{A}_{a, b}\right)} .
\end{aligned}
$$

The proof follows directly from the Cauchy formula for the Hardy space $H^{2}\left(\mathbb{D}_{c}\right)$ (see also Theorem 1 on p. 9 in [43]).

A nice feature of the kernel $K_{k}$ is that we have concise expressions: Obviously, we have

$$
K_{k}^{1}(z, \tau)=\left(\frac{z}{\tau}\right)^{k} \frac{1}{1-\left(\frac{z}{\tau}\right)^{2}}=\left(\frac{z}{\tau}\right)^{k} \frac{\tau^{2}}{\tau^{2}-z^{2}} .
$$


For $k$ odd we have the expressions

$$
\begin{array}{ll}
K_{k}^{2}(z, \tau)=\sum_{p=0}^{\infty}\left(\frac{z}{\tau}\right)^{2 p}=\frac{1}{1-\left(\frac{z}{\tau}\right)^{2}}=\frac{\tau^{2}}{\tau^{2}-z^{2}} \quad \text { for }|\tau|=b \\
K_{k}^{3}(z, \tau)=\sum_{p=1}^{\frac{d+k-2}{2}}\left(\frac{\tau}{z}\right)^{2 p}=-\left(1-\frac{\tau^{d+k-2}}{z^{d+k-2}}\right) \times K_{k}^{2} & \text { for }|\tau|=a .
\end{array}
$$

For $k$ even we have the expressions

$$
\begin{array}{ll}
K_{k}^{2}(z, \tau)=\sum_{p=0}^{\infty}\left(\frac{z}{\tau}\right)^{2 p+1}=\frac{z}{\tau} \frac{\tau^{2}}{\tau^{2}-z^{2}} & \text { for }|\tau|=b \\
K_{k}^{3}(z, \tau)=\sum_{p=1}^{\frac{d+k-2}{2}}\left(\frac{\tau}{z}\right)^{2 p}=-\frac{\tau}{z}\left(1-\frac{\tau^{d+k-2}}{z^{d+k-2}}\right) \times K_{k}^{2}(z, \tau) & \text { for }|\tau|=a
\end{array}
$$

The following estimates are important.

Theorem 5.2. For every $\varepsilon>0$ with $\varepsilon \leq(b-a) / 3$ and for $z$ and $\tau$ satisfying $a+\varepsilon<|z|<b-\varepsilon$ and $|\tau|=a$, or $|\tau|=b$, and for all $k \geq 0$ holds

$$
\left|K_{k}(z, \tau)\right| \leq C_{\varepsilon},
$$

where the constant $C_{\varepsilon}>0$ is independent of $k$.

The proof follows directly from the above expressions for the kernels $K_{k}^{j}, j=1,2,3$.

\section{Cauchy type kernel for the complexified annulus $\mathcal{A}_{a, b}$}

Finally, we are able to construct the Cauchy kernel for the complexified annulus $\mathcal{A}_{a, b}$.

First, we prove the following:

Proposition 6.1. Let $f \in H_{L}^{2}\left(\mathcal{A}_{a, b}\right)$. Then in $L_{2}$ sense the following limits hold true:

$$
\begin{aligned}
& \lim _{r \rightarrow a} f\left(r e^{i \varphi}, \theta\right)=\sum_{k, \ell} f_{k, \ell}^{*}\left(a e^{i \varphi}\right) Y_{k, \ell}(\theta) \\
& \lim _{r \rightarrow b} f\left(r e^{i \varphi}, \theta\right)=\sum_{k, \ell} f_{k, \ell}^{*}\left(b e^{i \varphi}\right) Y_{k, \ell}(\theta) .
\end{aligned}
$$

The proof mimics the classical proof in [40, Theorem 17.10.

Proof. First of all, we see that the function

$$
g\left(e^{i \varphi}, \theta\right)=\sum_{k, \ell} f_{k, \ell}^{*}\left(a e^{i \varphi}\right) Y_{k, \ell}(\theta)
$$


belongs to $L_{2}\left(\mathbb{S}^{1} \times \mathbb{S}^{d-1}\right)$. Indeed, we have the estimate

$$
\begin{aligned}
\int_{\mathbb{S}^{d}-1} \int_{0}^{2 \pi} & \left|\sum_{k, \ell} f_{k, \ell}^{*}\left(a e^{i \varphi}\right) Y_{k, \ell}(\theta)\right|^{2} d \theta d \varphi \leq \sum_{k, \ell}\left\|f_{k, \ell}^{*}\left(a e^{i \varphi}\right)\right\|_{L_{2}\left(\partial \mathbb{A}_{a, b}\right)}^{2} \\
& \leq \sum_{k, \ell}\left\|f_{k, \ell}\right\|_{H^{2}\left(\mathbb{A}_{a, b}\right)}^{2} \leq C \sum_{k, \ell}\left\|f_{k, \ell}\right\|_{H^{2}\left(\mathbb{A}_{a, b}\right)}^{2} L^{2 k} \\
& \leq C_{1}\|f\|_{H_{L}^{2}\left(\mathcal{A}_{a, b}\right)}^{2},
\end{aligned}
$$

where $C, C_{1}>0$. Similar estimate holds for $b$.

Let us put

$$
I_{r}:=\int_{\mathbb{S}^{d-1}} \int_{0}^{2 \pi}\left|\sum_{k, \ell} f_{k, \ell}^{*}\left(a e^{i \varphi}\right) Y_{k, \ell}(\theta)-\sum_{k, \ell} f_{k, \ell}\left(r e^{i \varphi}\right) Y_{k, \ell}(\theta)\right|^{2} d \theta d \varphi .
$$

We have to prove $I_{r} \longrightarrow 0$ for $r \longrightarrow a$. We have obviously

$$
I_{r} \leq \sum_{k, \ell} \int_{0}^{2 \pi}\left|f_{k, \ell}^{*}\left(a e^{i \varphi}\right)-f_{k, \ell}\left(r e^{i \varphi}\right)\right|^{2} d \varphi .
$$

We fix some $\varepsilon>0$. There exists a $k_{1} \geq 1$ such that for every $k \geq k_{1}$ holds

$$
\sum_{k=k_{1} \ell}^{\infty} \sum_{\ell=1}^{a_{k}}\left\|f_{k, \ell}\right\|_{H^{2}\left(\mathbb{A}_{a, b}\right)}^{2} L^{2 k}<\frac{\varepsilon}{2}
$$

This implies

$$
\sum_{k=k_{1}}^{\infty} \sum_{\ell=1}^{a_{k}} \int_{0}^{2 \pi}\left|f_{k, \ell}^{*}\left(a e^{i \varphi}\right)-f_{k, \ell}\left(r e^{i \varphi}\right)\right|^{2} d \varphi<C \frac{\varepsilon}{2}
$$

for some constant $C>0$. On the other hand, we find $\delta>0$ such that for $|r-a|<\delta$ holds

$$
\sum_{k=0}^{k_{1}-1} \sum_{\ell=1}^{a_{k}} \int_{0}^{2 \pi}\left|f_{k, \ell}^{*}\left(a e^{i \varphi}\right)-f_{k, \ell}\left(r e^{i \varphi}\right)\right|^{2} d \varphi<C \frac{\varepsilon}{2}
$$

Hence, we obtain $I_{r} \leq C \varepsilon$ which proves that $I_{r} \longrightarrow 0$ for $r \longrightarrow a$.

Recall that for every $f \in H_{L}^{2}\left(\mathcal{A}_{a, b}\right)$ all components $f_{k, \ell} \in H^{2, k}\left(\mathbb{A}_{a, b}\right)$ and they have limiting functions $f_{k, \ell}^{*} \in L_{2}\left(\partial \mathbb{A}_{a, b}\right)$. Hence, by Proposition 5.1 we obtain

$$
f(z, \theta)=\sum_{k, \ell}\left\langle K_{k}(z, \cdot), f_{k, \ell}^{*}(\cdot)\right\rangle_{H^{2}\left(\mathbb{A}_{a, b}\right)} Y_{k, \ell}(\theta) \quad \text { for all }(z, \theta) \in \mathcal{A}_{a, b} .
$$

Definition 6.2. We define the Cauchy type kernel $K$ for the space $H_{L}^{2}\left(\mathcal{A}_{a, b}\right)$ by putting

$$
K\left(z, \theta ; \tau, \theta^{\prime}\right)=\sum_{k=0}^{\infty} \sum_{\ell=1}^{a_{k}} \frac{1}{L^{k}} K_{k}(z, \tau) Y_{k, \ell}(\theta) Y_{k, \ell}\left(\theta^{\prime}\right)
$$


The function $K\left(z, \theta ; \tau, \theta^{\prime}\right)$ converges on compact subsets of $\mathcal{A}_{a, b}$ : Indeed, we use the estimates $\left|Y_{k, \ell}(\theta)\right| \leq C_{1} k^{\frac{d-2}{2}}$, and $a_{k} \leq C_{2} k^{d-2}$, cf. 45. By Theorem 5.2 we obtain the estimate

$$
\left|K\left(z, \theta ; \tau, \theta^{\prime}\right)\right| \leq C_{3, \varepsilon} \sum_{k=0}^{\infty} \frac{k^{d-2} k^{d-2}}{L^{k}}<\infty
$$

for all $a+\varepsilon \leq|z| \leq b-\varepsilon$ and $|\tau|=a$ or $|\tau|=b$.

Finally, we generalize the classical one-dimensional Cauchy formula, and justify the name "Cauchy type kernel" given to $K\left(z, \theta ; \tau, \theta^{\prime}\right)$ in Definition 6.2.

Theorem 6.3. The kernel $K\left(\zeta, \theta ; z, \theta^{\prime}\right)$ is a Cauchy type kernel for the space $H_{L}^{2}\left(\mathcal{A}_{a, b}\right)$ defined by the inner product (4.5), i.e. for every $f \in H_{L}^{2}\left(\mathcal{A}_{a, b}\right)$ holds

$$
\left\langle K\left(z, \theta ; z^{\prime}, \theta^{\prime}\right), f^{*}\left(z^{\prime}, \theta^{\prime}\right)\right\rangle_{H_{L}^{2}}=f(z, \theta), \quad \text { for all }(z, \theta) \in \mathcal{A}_{a, b},
$$

where $f^{*}$ is the boundary limit at $\partial \mathcal{A}_{a, b}$ of the function $f$.

The proof follows by a direct application of Theorem 5.2 .

\section{Error estimate of the Polyharmonic Gauss-Jacobi Cubature formula in the annulus}

The topic of estimation of quadrature formulas for analytic functions is a widely studied one. Beyond the classical monographs 31, [11, we provide further and more recent publications, as [6], 14, [15], [19, [27, [33. No references may be found though for the the Numerical Integration in the multivariate case, which is often called cubature formulas, even in the fundamental monographs as [47, 51], [48; see also the recent survey [9].

In order to explain our approach, first of all, we will recall the polyharmonic Gauss-Jacobi cubature formula presented in [21, 23. for the annulus $A_{a, b} \subset \mathbb{R}^{d}$. Let us fix some integers $N \geq 1$ and $k \geq 0$. We consider the following $2 N$-dimensional subspace of $V_{k, d}$ in (1.14),

$$
V_{k, d, N}:=\operatorname{span}\left\{\left\{r^{2 j+k}\right\}_{j=0}^{N-1},\left\{r^{-d-k+2+2 j}\right\}_{j=0}^{N-1}\right\} .
$$

We remark that this is a Chebyshev system of order $2 N$, cf. 30 .

Let the pseudo-positive (signed) measure $d \mu$ be given in the annulus $A_{a, b}$, see (1.5). For all indices $(k, \ell)$ the component measures are defined by

$$
d \mu_{k, \ell}(r):=\int_{\mathbb{S}^{d-1}} Y_{k, \ell}(\theta) d \mu(r \theta) \geq 0 \quad \text { for all } r \in[a, b] ;
$$

here the integral is symbolical with respect to the variables $\theta$. Rigorously, the component measure $d \mu_{k, \ell}(r)$ is defined for the functions $g(r)$ on the interval $[a, b]$ by means of the equality

$$
\int_{a}^{b} g(r) d \mu_{k, \ell}(r):=\int_{A_{a, b}} g(r) Y_{k, \ell}(\theta) d \mu(x) ;
$$

cf. [21], [23]. 
Let us fix $(k, \ell)$. Recall from [21], 23], that we consider truncated Moment problem and related Gauss-Jacobi type Quadrature formula corresponding to the operator $\Delta^{2 N}$. Thus, there exist points $t_{k, \ell ; j}, j=1,2, \ldots, 2 N$, belonging to the interval $[a, b]$, and non-negative numbers $\left\{\lambda_{k, \ell ; j}\right\}_{j=1}^{2 N}$, such that the following quadrature formula holds:

$$
\begin{aligned}
\int_{a}^{b} Q(t) d \mu_{k, \ell}(t) & =\int_{a}^{b} Q(t) d \mu_{k, \ell}^{G}(t) \\
& =\sum_{j=1}^{N} \lambda_{k, \ell ; j} Q\left(t_{k, \ell ; j}\right) \quad \text { for every } Q \in V_{k, d, 2 N}
\end{aligned}
$$

cf. [30], Theorem 4.1, chapter 4. We have put

$$
d \mu_{k, \ell}^{G}=\sum_{j=1}^{N} \lambda_{k, \ell ; j} \delta\left(t-t_{k, \ell ; j}\right)
$$

First of all, we will find the error for the above Quadrature formulas (7.2) following the classical scheme outlined in [11], p. $231-235$ (see also chapter 12 in [31]).

Let $H_{k, \ell ; 2 N}[f](t)$ be the unique element of $V_{k, d, N}$ which interpolates the function $f$ at the points $t_{k, \ell ; j}$, i.e.

$$
H_{k, \ell ; 2 N}[f]\left(t_{k, \ell ; j}\right)=f\left(t_{k, \ell ; j}\right) \quad \text { for } j=1,2, \ldots, 2 N .
$$

On the other hand, for every function $f \in H^{2, k}\left(\mathbb{A}_{a, b}\right)$ by Proposition 5.1 we have the Cauchy type formula

$$
f(z)=\frac{1}{2 \pi i} \int_{\partial \mathbb{A}_{a, b}} K_{k}(z, \tau) f^{*}(\tau) \frac{1}{\tau} d \tau,
$$

where $f^{*}$ is the limiting value of $f$ on the boundary $\partial \mathbb{A}_{a, b}$. Hence, we obtain

$$
H_{k, \ell ; 2 N}[f](z)=\frac{1}{2 \pi i} \int_{\partial \mathbb{A}_{a, b}} H_{k, \ell ; 2 N}\left[K_{k}(\cdot, \tau)\right](z) f^{*}(\tau) \frac{1}{\tau} d \tau
$$

here for every fixed $\tau$, by $H_{k, \ell ; 2 N}\left[K_{k}(\cdot, \tau)\right](z)$ we denote the element in $V_{k, d, N}$ which interpolates the kernel function $K_{k}(z, \tau)$ at $z=t_{k, \ell ; j}$ for $j=$ $1,2, \ldots, 2 N$. This gives us the remainder formula of Cauchy type for the interpolation (7.4),

$$
\begin{aligned}
f(z) & =H_{k, \ell ; 2 N}[f](z) \\
& +\frac{1}{2 \pi i} \int_{\partial \mathbb{A}_{a, b}}\left\{K_{k}(z, \tau)-H_{k, \ell ; 2 N}\left[K_{k}(\cdot, \tau)\right](z)\right\} f^{*}(\tau) \frac{1}{\tau} d \tau .
\end{aligned}
$$

Now we are able to estimate the error functional:

$$
E_{k, \ell}[f]:=\int_{a}^{b} f(t) d \mu_{k, \ell}(t)-\int_{a}^{b} f(t) d \mu_{k, \ell}^{G}(t) .
$$


Indeed, by (7.5) we have

$$
\begin{aligned}
& \int_{a}^{b} f(z) d \mu_{k, \ell}(z)=\int_{a}^{b} H_{k, \ell ; 2 N}[f](z) d \mu_{k, \ell}(z) \\
& \quad+\frac{1}{2 \pi i} \int_{\partial \mathbb{A}_{a, b}} \int_{a}^{b}\left\{K_{k}(z, \tau)-H_{k, \ell ; 2 N}\left[K_{k}(\cdot, \tau)\right](z)\right\} d \mu_{k, \ell}(z) f^{*}(\tau) \frac{1}{\tau} d \tau
\end{aligned}
$$

On the other hand, by (7.2) and (7.4) we obtain

$$
\begin{aligned}
\int_{a}^{b} H_{k, \ell ; 2 N}[f](z) d \mu_{k, \ell}(z) & =\int_{a}^{b} H_{k, \ell ; 2 N}[f](z) d \mu_{k, \ell}^{G}(z) \\
& =\int_{a}^{b} f(z) d \mu_{k, \ell}^{G}(z)
\end{aligned}
$$

which implies

$$
\begin{aligned}
E_{k, \ell}[f] & =\int_{a}^{b} f(t) d \mu_{k, \ell}(t)-\int_{a}^{b} f(t) d \mu_{k, \ell}^{G}(t) \\
& =\frac{1}{2 \pi i} \int_{\partial \mathbb{A}_{a, b}} \int_{a}^{b}\left\{K_{k}(z, \tau)-H_{k, \ell ; 2 N}\left[K_{k}(\cdot, \tau)\right](z)\right\} d \mu_{k, \ell}(z) f^{*}(\tau) \frac{1}{\tau} d \tau
\end{aligned}
$$

and we see that in the right-hand side we do not have derivatives of the function $f$ but only its values on the two circles $\partial \mathbb{A}_{a, b}$. Now we may prove the following Lemma.

Lemma 7.1. Let the knots $\left\{t_{k, \ell ; j}\right\}_{j=1}^{2 N}$ of the quadrature formula 7.2) lie in the interval $[a, b] \subset\left(a^{\prime}, b^{\prime}\right)$. Then for every function $f$ in the component Hardy space $H^{2, k}\left(\mathbb{A}_{a^{\prime}, b^{\prime}}\right)$, the error of the quadrature formula (7.2) satisfies

$$
\left|E_{k, \ell}[f]\right|=\left|\int_{a}^{b} f(t) d \mu_{k, \ell}(t)-\int_{a}^{b} f(t) d \mu_{k, \ell}^{G}(t)\right| \leq C_{k}\|f\|_{H^{2}\left(\mathbb{A}_{a^{\prime}, b^{\prime}}\right)} .
$$

Proof. Since $\left\{t_{k, \ell ; j}\right\}_{j=1}^{2 N} \in[a, b] \subset\left(a^{\prime}, b^{\prime}\right)$ it follows that the interpolation operator $H_{k, \ell ; 2 N}\left[K_{k}(\cdot, \tau)\right]$ depends continuously on $\tau \in \partial \mathbb{A}_{a^{\prime}, b^{\prime}}$. Hence, the function

$$
\int_{a}^{b}\left\{K_{k}(z, \tau)-H_{k, \ell ; 2 N}\left[K_{k}(\cdot, \tau)\right](z)\right\} d \mu_{k, \ell}(z)
$$

is also continuous in $\tau \in \partial \mathbb{A}_{a^{\prime}, b^{\prime}}$ and by Theorem 5.2 its modulus is bounded, namely

$$
\left|\int_{a}^{b}\left\{K_{k}(z, \tau)-H_{k, \ell ; 2 N}\left[K_{k}(\cdot, \tau)\right](z)\right\} d \mu_{k, \ell}(z)\right| \leq C_{k} \quad \text { for } \tau \in \partial \mathbb{A}_{a^{\prime}, b^{\prime}} .
$$

By application of Cauchy-Bunyakovksi-Schwarz inequality, from (7.7), we obtain for some constant $C>0$ the inequality

$$
\left|\int f(t) d \mu_{k, \ell}(t)-\int f(t) d \mu_{k, \ell}^{G}(t)\right| \leq C C_{k}\|f\|_{H^{2}\left(\mathbb{A}_{a^{\prime}, b^{\prime}}\right)}
$$

which ends the proof. 
Finally, we may proceed to the polyharmonic Gauss-Jacobi Cubature formula for the pseudo-positive measure $d \mu$. We will consider this in a simpler setting, for functions $f$ having a finite Laplace-Fourier expansion:

$$
f(x)=f(r \theta)=\sum_{k, \ell, k \leq k_{0}} f_{k, \ell}(r) Y_{k, \ell}(\theta) .
$$

The polyharmonic Gauss-Jacobi measure $d \mu^{G}(x)$ is defined as the pseudopositive measure having component measures (7.1) equal to $d \mu_{k, \ell}^{G}$ in (7.3). Written symbolically, we have the expansion

$$
d \mu^{G}(x)=\sum_{k, \ell} Y_{k, \ell}(\theta) d \mu_{k, \ell}^{G}(r) .
$$

The corresponding Cubature formula is simple to formulate if the function $f$ defined in $A_{a, b}$ has a finite expansion as in (7.9):

$$
\int_{A_{a, b}} f(x) d \mu^{G}(x)=\sum_{k, \ell, k \leq k_{0}} \int_{a}^{b} f_{k, \ell}(r) d \mu_{k, \ell}^{G}(r) .
$$

It is direct to see from the quadrature formulas (7.2), that for a function $u(x)$ which is a finite sum of the type (1.11) with $\Delta^{2 N} u(x)=0$ in $A_{a, b}$, holds

$$
\int_{A_{a, b}} u(x) d \mu(x)=\int_{A_{a, b}} u(x) d \mu^{G}(x),
$$

cf. 21], 23. This justifies the name polyharmonic cubature formula. Hence, we define the Cubature formula by putting

$$
C_{N}(f):=\int_{A_{a, b}} f(x) d \mu^{G}(x) .
$$

We will prove the estimate of the cubature formula in the following.

Theorem 7.2. Let $d \mu$ be a pseudo-positive measure, and $C_{N}(f)$ be the polyharmonic Gauss-Jacobi Cubature formula defined by 7.12) in the annulus $A_{a, b}$. Let $a^{\prime}<a$ and $b<b^{\prime}$, and we assume that the function $f \in H_{L}^{2}\left(\mathcal{A}_{a^{\prime}, b^{\prime}}\right)$ and has a finite representation (7.9) for some $k_{0} \geq 0$.

Then the error of the Cubature formula 17.11) is estimated by

$$
\begin{aligned}
|E[f]| & =\left|\int_{A_{a, b}} f(x) d \mu(x)-C_{N}(f)\right| \\
& \leq \sqrt{\sum_{k, \ell, k \leq k_{0}} \frac{C_{k}^{2}}{L^{2 k}} \times\|f\|_{H_{L}^{2}\left(\mathcal{A}_{a^{\prime}, b^{\prime}}\right)}^{2} .}
\end{aligned}
$$

Proof. Due to the finite expansion, we obtain the equality

$$
\int_{A_{a, b}} f(x) d \mu(x)=\sum_{k, \ell, k \leq k_{0}} \int_{a}^{b} f_{k, \ell}(r) d \mu_{k, \ell}(r) .
$$


On the other hand, by the Cubature formula (7.11) we have

$$
\int_{A_{a, b}} f(x) d \mu^{G}(x)=\sum_{k, \ell, k \leq k_{0}} \int_{a}^{b} f_{k, \ell}(r) d \mu_{k, \ell}^{G}(r) .
$$

Hence, we obtain the error functional

$$
\begin{aligned}
E[f] & =\int_{A_{a, b}} f(x) d \mu(x)-\int_{A_{a, b}} f(x) d \mu^{G}(x) \\
& =\sum_{k, \ell, k \leq k_{0}}\left(\int_{a}^{b} f_{k, \ell}(r) d \mu_{k, \ell}(r)-\int_{a}^{b} f_{k, \ell}(r) d \mu_{k, \ell}^{G}(r)\right)
\end{aligned}
$$

and by Lemma 7.1 we obtain

$$
\begin{aligned}
|E[f]| & =\left|\int_{A_{a, b}} f(x) d \mu(x)-\int_{A_{a, b}} f(x) d \mu^{G}(x)\right| \\
& \leq \sum_{k, \ell, k \leq k_{0}} C_{k}\left\|f_{k, \ell}\right\|_{H^{2}\left(\mathbb{A}_{a^{\prime}, b^{\prime}}\right)}=\sum_{k, \ell, k \leq k_{0}} \frac{C_{k}}{L^{k}}\left\|f_{k, \ell}\right\|_{H^{2}\left(\mathbb{A}_{a^{\prime}, b^{\prime}}\right)} L^{k} \\
& \leq \sqrt{\sum_{k, \ell, k \leq k_{0}} \frac{C_{k}^{2}}{L^{2 k}} \times\|f\|_{H_{L}^{2}\left(\mathcal{A}_{a^{\prime}, b^{\prime}}\right)}^{2} .}
\end{aligned}
$$

This ends the proof.

For practical purposes it is reasonable to work with functions having finite expansion (7.9). However, from Theorem 7.2 we see that the asymptotic behavior of the constants $C_{k}$ defined in (7.8) is important for the estimate of the error functional $E[f]$ for functions $f$ which do not have a finite expansion (7.9). This is a subtle task of Interpolation theory which is beyond the scope of the present paper.

Another important remark is that the cubature formula (7.12) may be easily extended, although in a non-unique manner, to signed measures $\mu$ having bounded variation. Indeed, we may represent the measure $\mu$ as a difference, $\mu=\mu^{1}-\mu^{2}$, where $\mu^{1}, \mu^{2}$ have bounded variation. We can do this representation componentwise (non-uniquely) by $\mu_{k, \ell}=\mu_{k, \ell}^{1}-\mu_{k, \ell}^{2}$ with measures $d \mu_{k, \ell}^{1}, d \mu_{k, \ell}^{2} \geq 0$. Then we find the polyharmonic Gauß-Jacobi cubature measures $\mu^{1, G}$ and $\mu^{2, G}$ and put $C_{N}(f)=\int f d \mu^{1, G}-\int f d \mu^{2, G}$ for the cubature formula.

Our research may be considered as a contribution to the topic of analytic continuation of solutions to elliptic equations (in particular, harmonic functions), see the discussion and references to the works of V. Avanissian, P. Lelong, C. Kiselman, J. Siciak, M. Jarnicki, T. du Cros, on p. $54-55$ in [3, 4], 34], 13, 22, 25]. On the other hand, the concept of polyharmonic Hardy spaces which we introduce appears to be a new multivariate concept of Hardy space which differs from the existing approaches, cf. [49], [50], 41], 
42, 8], 44], 46. This notion will be given a thorough study in a planned monograph 24. Another setting where the polyharmonic Gauss-Jacobi cubature formulas and Hardy space $H_{L}^{2}$ may be naturally developed, is the strip $[a, b] \times \mathbb{R}^{d}$, cf. [21], 24].

\section{Acknowledgment}

Both authors thank the Alexander von Humboldt Foundation.

\section{References}

[1] G.E. Andrews, R. Askey, R. Roy, Special functions. Encyclopedia of Mathematics and its Applications, 71. Cambridge University Press, Cambridge, 1999.

[2] D. H. Armitage, M. Goldstein, Quadrature and Harmonic $L^{1}$-Approximation in Annuli, Trans. Amer. Math. Soc. 312 (1989), 141-154.

[3] N. Aronszajn, T.M. Creese, L.J. Lipkin, Polyharmonic Functions, Clarendon Press, Oxford 1983.

[4] V. Avanissian, Cellule d'harmonicité et prolongement analytique complexe, Hermann, Paris, 1985.

[5] S. Axler, P. Bourdon, W. Ramey, Harmonic Function Theory, second edition, Springer, New York, 2001.

[6] N. S. Bakhvalov, On the optimal speed of integrating analytic functions, U.S.S.R. Comput. Math. Math. Phys.7 (1967), 63-75.

[7] S. Bergman, The Kernel Function and Conformal Mapping, Amer. Math. Soc., Providence, RI, 1970.

[8] R. Coifman, G. Weiss, Extensions of Hardy spaces and their use in analysis, Bull. Amer. Math. Soc. Volume 83, Number 4 (1977), 569-645.

[9] R. Cools, An Encyclopaedia of Cubature Formulas, J. Complexity, 19 (2003), $445-453$.

[10] P. Davis, Interpolation and Approximation. Dover Publications Inc., New York, 1975.

[11] P. Davis, P. Rabinowitz, Methods of Numerical Integration. Second edition. Computer Science and Applied Mathematics. Academic Press, Inc., Orlando, FL, 1984.

[12] P. Dirac, Wave equations in conformal space, Ann. of Math., 37 (1936), no. 2, 429-442.

[13] K. Fujita, M. Morimoto, On the double expansion of holomorphic functions, J. Math. Anal. Appl. 272 (2002), 335-348.

[14] W. Gautschi, R.S.Varga, Error Bounds for Gaussian Quadrature of Analytic Functions, SIAM J. Numer. Anal. 20, p. 1170-1186 (1983).

[15] M. Goetz, Optimal quadrature for analytic functions, J. Comput. Appl. Math. 137 (2001), 123-133.

[16] M. Goldstein, W. Haußmann, L. Rogge, A harmonic quadrature formula characterizing bi-infinite cylinders, Michigan Math. J., 42 (1995), 175-191.

[17] K. Hoffman, Banach spaces of analytic functions, Prentice Hall, Englewood Cliffs, New Jersey, 1962. 
[18] N. Kerzman, E. M. Stein, The Cauchy Kernel, the Szegö Kernel, and the Riemann Mapping Function, Math. Ann. 236 (1978), 85-93.

[19] M. Kzaz, Convergence acceleration of some Gaussian quadrature formulas for analytic functions, J. Appl. Numer. Math. 10 (1992), 481-496.

[20] O. Kounchev, Multivariate Polyspnes. Applications to Numerical and Wavelet Analysis, Academic Press, San Diego, 2001.

[21] O. Kounchev, H. Render, Reconsideration of the multivariate moment problem and a new method for approximating multivariate integrals, electronic version at arXiv:math/0509380v1 [math.FA]

[22] O. Kounchev, H. Render, Holomorphic Continuation via Laplace-Fourier series, Contemporary Mathematics 455 (2008), 197-205.

[23] O. Kounchev, H. Render, The moment problem for pseudo-positive definite functionals, Arkiv for Matematik, 48 (2010), 97-120.

[24] O. Kounchev, H. Render, Multivariate Moment Problem, Hardy Spaces, and Orthogonality, in preparation.

[25] O. Kounchev, H. Render, Polyharmonic functions of infinite order on annular regions, submitted.

[26] O. Kounchev, H. Render, Polyharmonic Hardy spaces on the Klein-Dirac quadric, in preparation.

[27] M. A. Kowalski, A. G. Werschulz, and H. Wozniakowski, Is Gauss quadrature optimal for analytic functions?, Numerische Mathematik, Volume 47, Number 1 / March, 1985, 89-98.

[28] S. Krantz, Explorations in Harmonic Analysis, Springer, Berlin, 2009.

[29] S. Krantz, Geometric Function Theory, Birkhäuser, Basel, 2005.

[30] M. Krein, A. Nudelman, The Markov moment problem and extremal problems, Amer. Math. Soc., Providence, R.I., 1977.

[31] V. Krylov, Approximate calculation of integrals. Translated by Arthur $H$. Stroud, The Macmillan Co., New York-London, 1962.

[32] S. McCullough, Li-Chien Shen, On the Szegö kernel of an annulus, Proc. Amer. Math. Soc. 121 (1994), 1111-1121.

[33] G. Milovanovic, M.M. Spalevic, Error bounds for Gauss-Turán quadrature formulae of analytic functions, Math. Comp. 72 (2003), 1855-1872.

[34] M. Morimoto, Analytic Functionals on the Sphere, Translation of Mathematical Monographs, Vo. 178, Amer. Math. Soc., Providence, Rhode Island 1998.

[35] N. Nikolov, I. Todorov, Conformal Quantum Field Theory in Two and Four Dimensions, In: Proceedings of the Summer School in Modern Mathematical Physics, eds. B. Dragovich, B. Sazdović, Belgrade 2002, 1-49; online available at arxiv.

[36] N.M. Nikolov, I.T. Todorov, Conformal invariance and rationality in an even dimensional quantum field theory, Int. J. Mod. Phys. A19 (2004) 3605-3636; math-ph/0405005

[37] N. Nikolov, I. Todorov, Lectures on Elliptic Functions and Modular Forms in Conformal Filed Theory, math-ph/ 0412039

[38] R. Penrose, On the Origins of Twistor Theory, In: Gravitation and Geometry, a volume in honour of I. Robinson, Biblipolis, Naples 1987; online in http://users.ox.ac.uk/ ${ }^{\sim}$ web/00001/ 
[39] R.M. Range, Holomorphic Functions and Integral Representations in Several Complex Variables, Springer, Berlin, 1986.

[40] W. Rudin, Real and Complex Analysis, McGraw-Hill, New York, 1976.

[41] W. Rudin, Function theory in polydiscs, Benjamin, Inc., New York, 1969.

[42] W. Rudin, Function Theory in the Unit Ball of $C^{n}$, Springer-Verlag, NewYork, 1980.

[43] D. Sarason, The $H^{p}$ spaces of an annulus, Memoirs of AMS, Number 56, 1965.

[44] D. Sarason, Holomorphic Spaces: A Brief and Selective Survey, In: Holomorphic Spaces, MSRI Publications, Cambridge University Press, Cambridge, vol. 33, 1998.

[45] R. Seeley, Spherical harmonics, Amer. Math. Monthly, 73 (1966), 115-121.

[46] B.A. Shaimkulov, On holomorphic extendability of functions from part of the Lie sphere to the Lie ball, Siberian Math. J. 44 (2003), 1105-1110.

[47] S.L. Sobolev, Cubature formulas and modern analysis. An introduction. Translated from the 1988 Russian edition. Gordon and Breach Science Publishers, Montreux, 1992.

[48] S. Sobolev, V. Vaskevich, The theory of cubature formulas, Springer, Berlin, 1997.

[49] E.M. Stein, Singular Integrals and Differentiability Properties of Functions, Princeton University Press, 1970.

[50] E.M. Stein, G. Weiss, Introduction to Fourier Analysis on Euclidean spaces, Princeton University Press, 1971.

[51] A.H. Stroud, Approximate calculation of multiple integrals, Prentice-Hall, Englewood Cliffs, N.J., 1971.

[52] A.H. Stroud, Numerical quadrature and solution of ordinary differential equations. Springer-Verlag, New York-Heidelberg, 1974.

[53] A. N. Tikhonov, A. A. Samarskii, Equations of Mathematical Physics, Dover Publications, 1990.

[54] I. Vekua, New Methods for Solving Elliptic Equations. Wiley, New York, 1967.

Ognyan Kounchev

Acad. G. Bonchev, bl. 3

1113 Sofia

Bulgaria

and IZKS, University of Bonn

e-mail: kounchev@math.bas.bg

Hermann Render

School of Mathematical Sciences

Belfield

Dublin 4

Ireland

e-mail: hermann.render@ucd.ie 